\title{
Load Balancing Routing in Low-Cost Parallel QoS Sensitive Network Architecture
}

\author{
Furong Wang, Ye Wu \\ Huazhong University of Science and Technology, \\ Wuhan, Hubei, China \\ Wangfurong@hust.edu.cn \\ Minibeepcn@yahoo.com.cn
}

\begin{abstract}
A low-cost parallel QoS Sensitive domain, which supports load balancing network architecture, is developed in the paper. To deal with the scaling problem, a large network, thus, is structured by grouping nodes into parallel domains. Different from traditional approaches, especially hierarchical structure, parallel structure aggregates the topologies of domains in a new way. The corresponding routing algorithm, which adopts two skills for low-cost routing, QoS line segment and swapping matrix, is to emphasize particularly on load balancing within networks. Finally, Simulation results show appealing performances in terms of different metrics.
\end{abstract}

\section{Introduction}

QoS Routing is a process for the purpose of finding a path from the source node to the destination node and satisfying end-system performance requirements. Routing messages of a multi-metric QoS routing algorithm, consume enormous amount of network resources. For instance, an algorithm in [6] for finding a route with additive QoS constraints is proposed, but its price for broadcasting routing messages is scarcely taken into account. Meanwhile, the seriousness of load balancing is intended to cause a prodigious waste and congestion partially or full-scaly. QoS routing based on parallel-domain network, better than traditional aggregation suggested in PNNI[1], effectually reduce the outburst of unbalanced traffic load by ameliorating route computation versus QoS provisioning and route computation versus load balancing.

\section{Network Architecture and Domain Models}

A domain is modeled as a tuple $(N, M, E)$, where $N$ is the set of nodes, $M \subset N$ is the set of border nodes, and $E$ is the set of physical links. The QoS parameter of a physical link is denoted as a QoS pair $(D, B)$, in which $D$ is the best delay of the link and $B$ is the residual bandwidth. If a path is made up of $n-1$ physical links, each of which is characterized with a QoS pair $\left(D_{k}, B_{k}\right)$, the QoS pair of a physical path is denoted as: 


$$
\left(D^{\prime}, B^{\prime}\right)=\left(\sum_{k=0}^{n-1} D_{k}, \min _{k} B_{k}\right)
$$

When there exist $m(m>1)$ paths between two nodes, a QoS pair set like $\left\{\left(D_{0}^{\prime}, B_{0}^{\prime}\right),\left(D_{1}^{\prime}, B_{1}^{\prime}\right) \ldots\left(D_{m}^{\prime}, B_{m}^{\prime}\right)\right\}$ must be used to denote QoS information between two nodes. A network, denoted as $(G, L)$ consists of a set of domains and joint links, where $G=\left\{g_{i}=\left(N_{\mathrm{i}}, M_{\mathrm{i}}, E_{\mathrm{i}}\right), 1 \leq i \leq|G|\right\} . L$ is the set of inter-domain links, each of which is denoted in the same way as the intra-domain links in a domain.

\section{QoS Sensitive Aggregations}

Fig. 1 shows two line segments of a physical path between two nodes denoted by [low_left, high_right] at time $\mathrm{T}_{1}$ and $\mathrm{T}_{2}$. Different line segments would have different crankback and err-denial. Similarly, the QoS approximation of a logical interdomain path can also be denoted by a line segment such as $l_{\text {path }}$, which must include three essential parts, the originating node and domain, the outgoing/incoming border node and domain, and the current incoming/outgoing border node and domain.

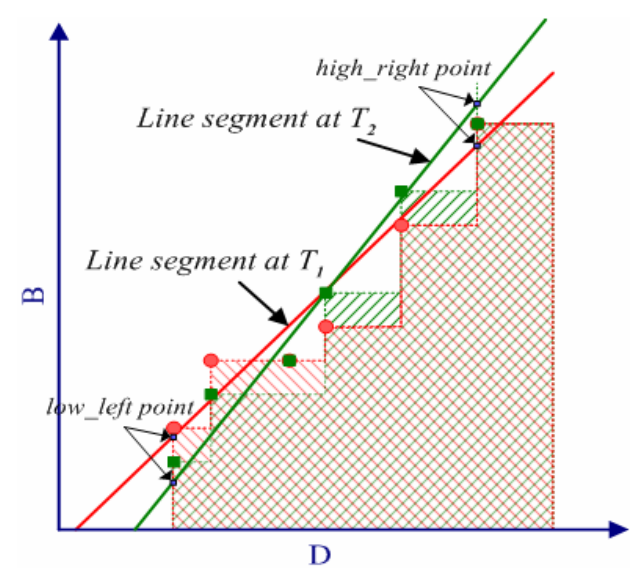

Fig. 1. A line Segment are generated by the least square method applied on its QoS pair set. The shift of line segments may occur with the process of time. Route requests in the unshaded area between the line segment and the staircase can not be served at all, which is called crankback that is caused by the distortion of line segment. Meanwhile, it would reject feasible requests as it doesn't cover some areas that belong to staircase, which is called err-denial

A line segment can be evaluated for its approximate residual traffic load on the path. For a line segment $\left[\left(\mathrm{D}_{0}, \mathrm{~B}_{0}\right),\left(\mathrm{D}_{1}, \mathrm{~B}_{1}\right)\right]$, its residual traffic load is defined as:

$$
B_{\text {path }}=\left\{\int_{D_{0} \mathrm{~L}}^{D_{\mathrm{L}}}\left[\frac{\left(B_{1}-B_{0}\right) D}{\left(D_{1}-D_{0}\right)}+\frac{\left(B_{0} D_{1}-B_{1} D_{0}\right)}{\left(D_{1}-D_{0}\right)}\right]\right\} /\left(D_{1}-D_{0}\right)=\frac{B_{0}+B_{1}}{2}
$$

There are totally $\lambda$ outgoing border nodes $\mathrm{w}, \mathrm{x} \ldots \mathrm{z}$ within domain $\mathrm{Y}$ adjacent to domain $\mathrm{Z}$ and node $\mathrm{i}$ is the current incoming border node within domain $\mathrm{Z}$, or there are totally $\lambda$ incoming border nodes $\mathrm{w}, \mathrm{x} \ldots \mathrm{Z}$ within domain $\mathrm{Y}$ and node $\mathrm{i}$ is the cur- 
rent outgoing border node within domain $\mathrm{Y}$. When a route request originates from node o within domain $\mathrm{X}$, the swapping matrix for node $\mathrm{i}$ is defined as follows:

$$
\left[\begin{array}{c}
l_{\text {owi }} \\
l_{\text {oxi }} \\
\vdots \\
l_{\text {ozi }}
\end{array}\right]_{\lambda \times 1}=\left[\begin{array}{cc}
\left(D_{\text {low_left }}, B_{\text {low_left }}\right)_{\text {owi }} & \left(D_{\text {high_right }}, B_{\text {high_right }}\right)_{\text {owi }} \\
\left(D_{\text {low_left }}, B_{\text {low_left }}\right)_{o x i} & \left(D_{\text {high_right }}, B_{\text {high_right }}\right)_{o x i} \\
\vdots & \vdots \\
\left(D_{\text {low_left }}, B_{\text {low_left }}\right)_{o z i} & \left(D_{\text {high_right }}, B_{\text {high_right }}\right)_{o z i}
\end{array}\right]_{\lambda \times 4}
$$

\section{Line-Segment and Balance-Swapping Routing Algorithm}

A problem with two constraints called "shortest weight-constrained path" was listed in [4]. Furthermore, as a four-metric routing algorithm, $L B R A$ 's nature is close to the centralized bandwidth-delay routing algorithm $(C B D R A)$ as in [3]. Like [5], LBRA prefers source routing mechanism to Hop-by-Hop routing mechanism because it needs the topological border information to analyze resource allocations. There are totally two conjoint levels in $L B R A$, inter-domain routing level and intra-domain routing level. Intra-domain routing level is analogous to $C B D R A$ if residual traffic load is not taken into account. Suppose that there is a route request from node $o$ with the QoS pair $\left(d_{r e q}, b_{\text {req }}\right)$. Let $D_{i}, D_{i+1} \ldots D_{i+n}$ be the estimated sum of delay from the source node to outgoing border node $i, i+1 \ldots i+n$ within the same domain, which incoming node $k$ doesn't lie in. The link between node $i$ and node $k$ is an inter-domain $\operatorname{link}_{i k}\left(D_{i k}, B_{i k}\right)$. So is the link between node $i+1 \ldots$ and node $k$.

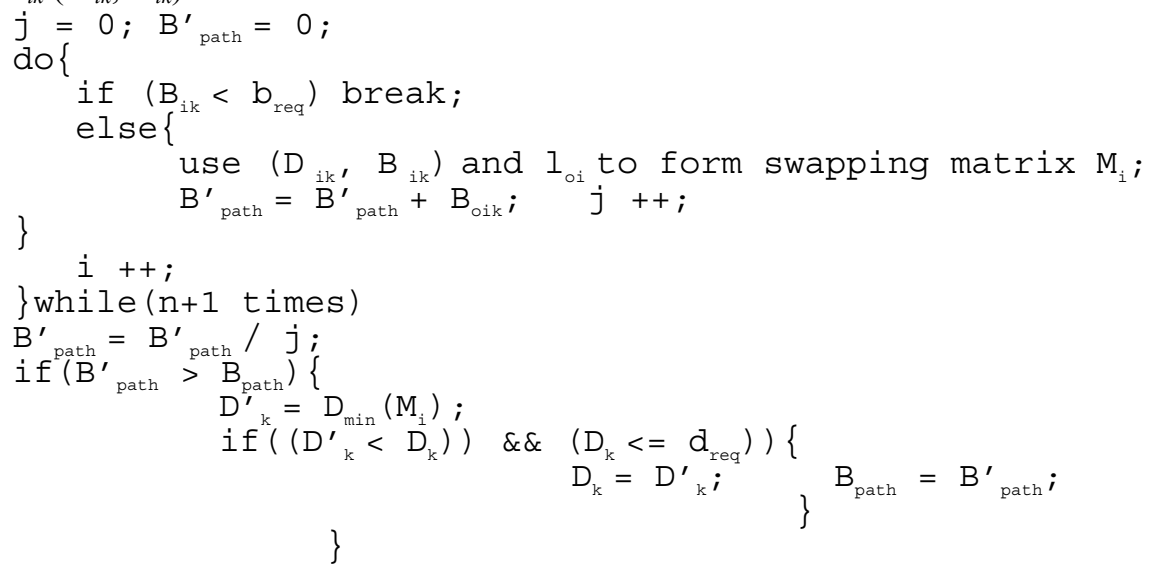

An outgoing node runs LBRA similar to an incoming node, except that the out going node use $\left[\left(D_{i k}, B_{i k}\right),\left(D^{\prime}{ }_{i k}, B^{\prime}{ }_{i k}\right)\right]$ and $1_{o i}$ to form a swapping matrix $M_{i}$.

\section{Simulation Results}

The simulated network consists of 9 domains with a total of 253 nodes. The number of borders varies from 4 to 6 . All nodes are connected by directed links and each node is connected to at least 2 other nodes in the same domain. The delay of each link 
is between $2 \mathrm{~ms}$ to $15 \mathrm{~ms}$ and the bandwidth ranges from 640K to 6.4M Bytes/s. Fig. 2 reflects routing success ratio w.r.t. bandwidth and link utilization.
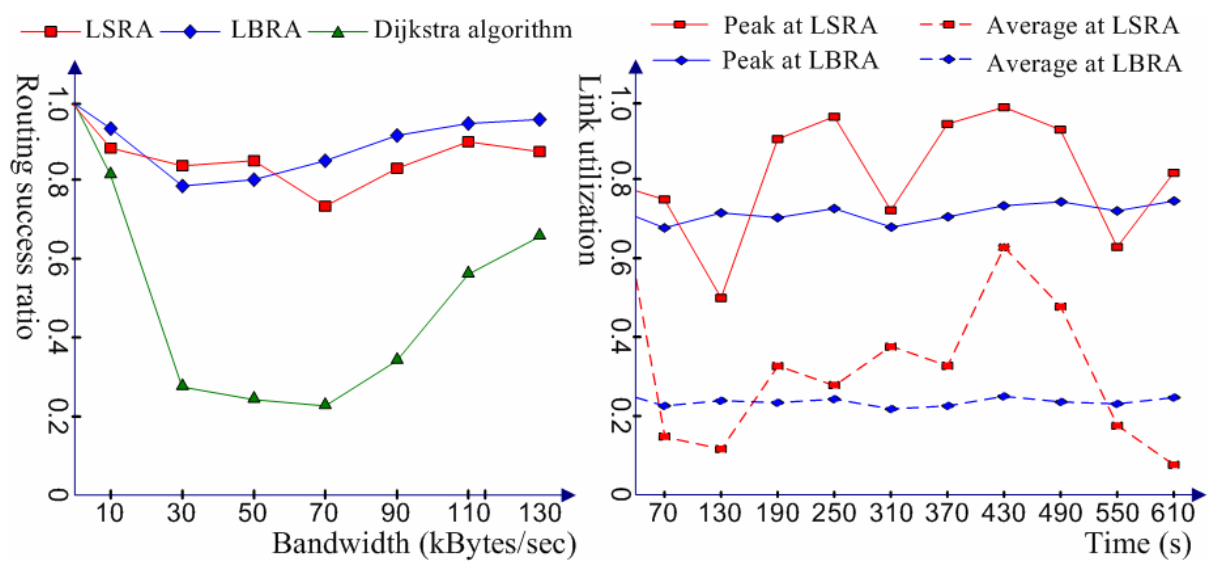

Fig. 2. In the left sub-figure, LBRA outperforms Dijkstra algorithm and LSRA as in [2] in routing success ratio evaluation w.r.t bandwidth. In the right sub-figure, LBRA doesn't cause any congestion and keep the smooth trend of link utilization on the random link, while LSRA fails to obviate the outburst of unbalanced traffic load

\section{Conclusions}

In the paper, I use line segments and swapping matrix to achieve a load-balancing QoS sensitive network and develop LBRA which can find the most appropriate QoS route. Simulations show a stable and high performance of different metrics.

\section{References}

1. The ATMForum: Private Network-to-network Interface Specification Version 1.0 (pnni 1.0). fpnni-0055.000 (1996)

2. King-Shan Lui, Klara Nahrstedt: Topology Aggregation and Routing in Bandwidth-delay Sensitive Networks. In Proceedings of GLOBECOM '00. IEEE (2000)

3. Z. Wang, J. Crowcroft: Quality-of-service Routing for Supporting Multimedia Applications. In IEEE Journal on Selected Areas in Communications. Vol.14. (1996)

4. M. R. Garey, D. S. Johnson: Computers and Intractability - A Guide to the Theory of NPCompleteness. Freeman, California, USA (1979)

5. D. Estrin, Y. Rekhter, and S. Hotz: Scalable Inter-Domain Routing Architecture. In Proceedings of ACM SIGCOMM'92, Maryland (1992)

6. Turgay Korkmaz, Marwan Krunz, and Spyros Tragoudas: An Efficient Algorithm for Finding a Path Subject to Two Additive Constraints. In Computer Communications Journal, Vol. 25. (2002) 225-238 